\title{
Localized overheating on aluminum metafilms mediated by surface plasmons
}

\author{
D. E. Martínez-Lara ${ }^{a, *}$, R. González-Campuzano ${ }^{a, c}$, J. L. Benítez ${ }^{b}$ and D. Mendoza ${ }^{a, * *}$ \\ ${ }^{a}$ Instituto de Investigaciones en Materiales, Universidad Nacional Autónoma de México, \\ Apartado Postal 70-360, Ciudad de México 04510, México. \\ e-mail:*davideduardo@ciencias.unam.mx; ${ }^{* *}$ doroteo@unam.mx \\ ${ }^{b}$ Instituto de Ciencias Aplicadas y Tecnología, Universidad Nacional Autónoma de México, \\ Apartado Postal 70-186, Ciudad de México 04510, México. \\ ${ }^{c}$ Current affiliation is $b$
}

Received 18 February 2021; accepted 10 April 2021

\begin{abstract}
We studied the increase in temperature of systems formed by thin aluminum films deposited on texturized substrates which we denominated aluminum metafilms. By varying the geometric parameters of the metafilms, surface plasmons in the wavelength range of $\sim 420-770 \mathrm{~nm}$ were excited. Temperature measurements as a function of the intensity of incident radiation in the interval from 0 to $4 \times 10^{18}$ photons/(s) $\mathrm{cm}^{2}$ using wavelengths of 445, 532 and $650 \mathrm{~nm}$, showed temperature increases up to $\sim 200 \mathrm{~K}$, these attributed to metafilm morphology and hot electrons result of the non-radiative decay of the surface plasmons. Also, increases from $\sim 1.3 \times 10^{-6} \Omega \mathrm{cm}$ to $2.3 \times 10^{-2} \Omega \mathrm{cm}$ in the electrical resistivity were recorded, when the metafilms were irradiated for times of $1 \mathrm{~s}$; when the exposure times were greater than $4 \mathrm{~s}$, irreversibly changes in the morphology of the samples were observed.
\end{abstract}

Keywords: Surface Plasmons; Metamaterials; Aluminum; nanostructures.

PACS: 73.20.Mf; 81.05.Xj; 78.67.Pt; 73.50.-h; 68.60.Dv

DOI: https://doi.org/10.31349/RevMexFis.67.051001

\section{Introduction}

Surface plasmons (SP) are collective oscillations of charge located between the surface of a dielectric and a metal [1], which have made it possible to improve and reduce the dimensions of electronic devices [2-4]. In biomedicine, they are being used in photothermal therapies and cancer treatments $[5,6]$, as well as their use to convert solar energy into chemical energy [7]. Recent studies have shown that systems formed by plasmonic nanostructures can be used as nano heat sources $[8,9]$.

Among the traditionally used metals in plasmonics, silver $(\mathrm{Ag})$ and gold $(\mathrm{Au})$ stand out, because they have a wide range of the electromagnetic spectrum in which SP can be excited, ranging from infrared (IR) to visible (Vis) with energy losses at $\sim 318 \mathrm{~nm}$ and $\sim 517 \mathrm{~nm}$ as a result of their interband transitions (IT) $[10,11]$ respectively.

On the other hand, aluminum ( $\mathrm{Al})$ is a metal that has begun to be explored for plasmonic applications [12,13], which in comparison with $\mathrm{Ag}$ and $\mathrm{Au}$, has some advantages, such as a broader SP excitation range that goes from ultraviolet (UV) to IR; it has a layer of native self-limited oxide $\sim 3-4 \mathrm{~nm}$ [14] that protects it from corrosive environments, as well as being cheaper. Due to its high density energy, it can release large amounts of energy through the SP-assisted combustion process (a phenomenon known as photo-ignition), which can be applied for the development of nanosolders, nano propulsures or nanoexplosives [15].

Zheng et al. [16] have shown that hot carriers (hot electrons) induced by plasmons, can be used in two-dimensional structures to generate photocurrents [17], with the capacity to transfer their energy to phonons [5], producing temperature increases in nanometric areas in a matter of femtoseconds [18].

It has recently been shown that it is possible to excite SP at certain wavelengths by varying the metal nanostructures dimensions of $\mathrm{Al}, \mathrm{Pb}$, and $\mathrm{Ag}$ [19-21]. In this work, $\mathrm{Al}$ thin films have been deposited on nanostructured substrates called aluminum metafilms (Al-MF), obtained by electrochemical anodization. By varying the dimensions of the Al-MF, it was possible to excite SP at determined wavelengths in the range $\sim 420-770 \mathrm{~nm}$. Increases in temperature $(\mathrm{T})$ and changes in electrical resistivity $(\rho)$ of Al-MF were recorded when irradiated with different wavelengths.

\section{Experimental details}

For the synthesis of the nanostructured substrates, $\mathrm{Al}$ foils (Sigma Aldrich, $0.25 \mathrm{~mm}$ thick, $99.999 \%$ purity) were used, which were annealed in an $\mathrm{H}_{2}$ atmosphere at $600^{\circ} \mathrm{C}$ for $6 \mathrm{hrs}$. Subsequently, they were electropolished in an electrochemical cell using as electrolyte a solution of perchloric acid and ethanol in a ratio of 5:1 vol., applying voltages of $18 \mathrm{~V}$ for 2 min maintaining a temperature of $\sim 3^{\circ} \mathrm{C}$, in this way, it is possible to obtain a homogeneous surface like the one shown in Fig. 1b).

In the anodization process graphite was used as the cathode and $\mathrm{Al}$ as the anode. The anodizing voltages were in the range of 130-250 V supplied by an adjustable DC power source (BK PRECISION model 9185B). The electrolytes used were chosen depending on the applied anodizing voltage [19]. 

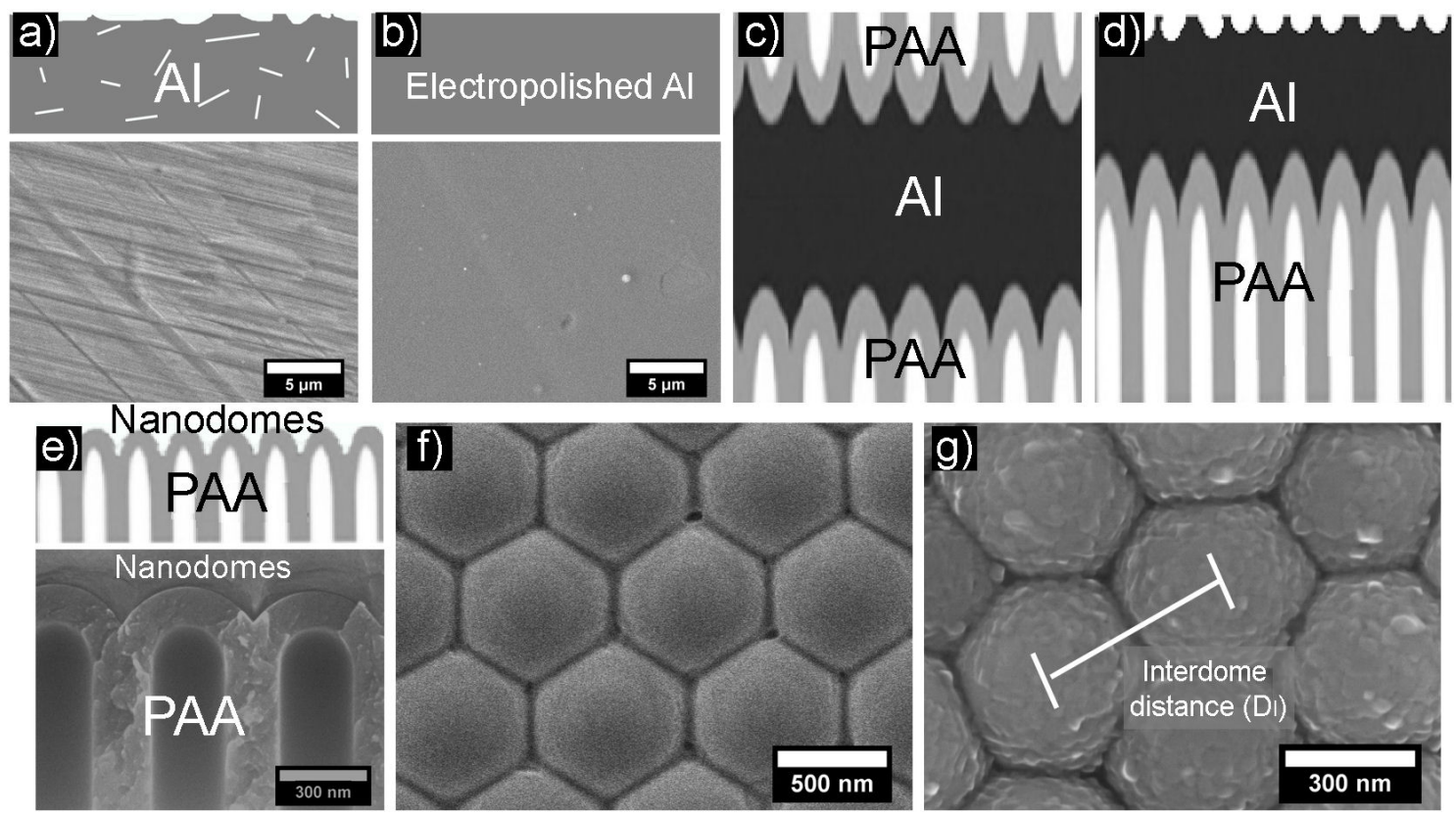

FIGURE 1. Schematic representation and SEM micrographs of the Al-MF synthesis process. a) Al foil without treatment. b) Electropolished Al foil. c) PAA obtained after the anodization process. d) Elimination of the PAA. e) Cross-section and f) Top view of the nanostructured substrates (nanodomes). g) Al film deposited on a nanostructured substrate (Al-MF).

From the anodization process, a type of $\mathrm{Al}$ oxide known as Porous Anodic Alumina (PAA) was obtained (see Fig. 1c)). Subsequently, using a solution of $\mathrm{NaOH}(2.5 \mathrm{M})$ and $\mathrm{HCl}(20 \%$ weight), the PAA was eliminated from one of the faces of the foil; afterward, the residual $\mathrm{Al}$ was removed with a solution of $\mathrm{CuCl}_{2}(0.5 \mathrm{M})$ (Fig. 1d)). With this process, the nanostructured substrate (nanodomes), shown in Figs. 1e) and 1f), is obtained.

On the nanodomes (Fig. 1f)) a thin film of $\mathrm{Al}$ of $\sim 50 \mathrm{~nm}$ thick was deposited by thermal evaporation using pressures of $\sim 5 \times 10^{-6}$ torr, thus obtaining what we denominated Al-MF (Fig. 1g)). Its morphology was evaluated using a field emission scanning electron microscope (SEM) JEOL 7600F equipped with an energy dispersive $\mathrm{x}$-ray spectrometer (EDS). The geometric parameters of the Al-MF, such as the interdome distance $\left(D_{I}\right)$ (Fig. 1g)) were obtained using the Image J software [22]. After, employing a UV-Vis spectrophotometer (UV-2600 Shimadzu Corporation), the specular reflectance measurements were performed at an angle of incidence of $8^{\circ}$ in a range of $190-1400 \mathrm{~nm}$.

The measurements of $T$ as a function of the intensity of incident radiation were carried out at a pressure of $\sim 5 \times 10^{-3}$ torr, using a K-type thermocouple of $\sim 0.2 \mathrm{~mm}$ diameter attached to the sample using silver paint (Colloidal silver paste-EMS). The samples were irradiated in an area of $\sim 0.8$ $\mathrm{mm}^{2}$ using monochromatic CW laser light sources of 445 , 532 and $650 \mathrm{~nm}$, varying the incident intensity in an interval of $0-4 \times 10^{18}$ photons/(s) $\mathrm{cm}^{2}$ (Maximum power of the lasers). Finally, measurements of $\rho$ as a function of $T$ were carried out in the range of $\sim 100-370 \mathrm{~K}$, using the four-terminal sensing, in darkness and under an intensity of
$1 \times 10^{16}$ photons $/(\mathrm{s}) \mathrm{cm}^{2}$ using the three wavelengths. For these measurements, the maximum light intensity was chosen monitoring the electrical resistance in situ while increasing the intensity and was stopped when we detected a minimum change; with this procedure we think the overheating is minimized.

\section{Results and discussion}

In Fig. 2a), an SEM micrograph of an $\mathrm{Al}$ film deposited on a non-texturized substrate (glass) is shown, while in Figs. 2b) and 2c) Al films deposited on texturized substrates (nanodomes) are shown. In these last two cases, it can be seen that the $\mathrm{Al}$ film follows the morphology of the substrate.

The values of the interdome distance $\left(D_{I}\right)$ obtained from the SEM images (Fig. 1g)) were in the range of $415.5 \pm 57.8-$ $786.9 \pm 90.3 \mathrm{~nm}$. The $D_{I}$ value depends on the applied anodizing voltage [19-21].

In Fig. 3a) the reflectance spectra as a function of the wavelength of Al-MF with different $D_{I}$ values are shown. In all cases, minima are observed in the $\sim 420-770 \mathrm{~nm}$ wavelength range attributed to first-order surface plasmon resonances (SPR) [1]. The position of these minima can be calculated using as a first approximation the electromagnetic wave coupling model and SPR in two-dimensional periodic arrays with hexagonal geometry, given by [24]:

$$
\begin{aligned}
\frac{\varepsilon_{m}(\lambda) \varepsilon_{d}}{\varepsilon_{m}(\lambda)+\varepsilon_{d}} & =\sin ^{2} \theta+\frac{2}{\sqrt{3}} \frac{\lambda}{D_{I}}(2 i+j) \sin \theta \\
& +\frac{4}{3} \frac{\lambda^{2}}{D_{I}^{2}}\left(i^{2}+j^{2}+i j\right)
\end{aligned}
$$



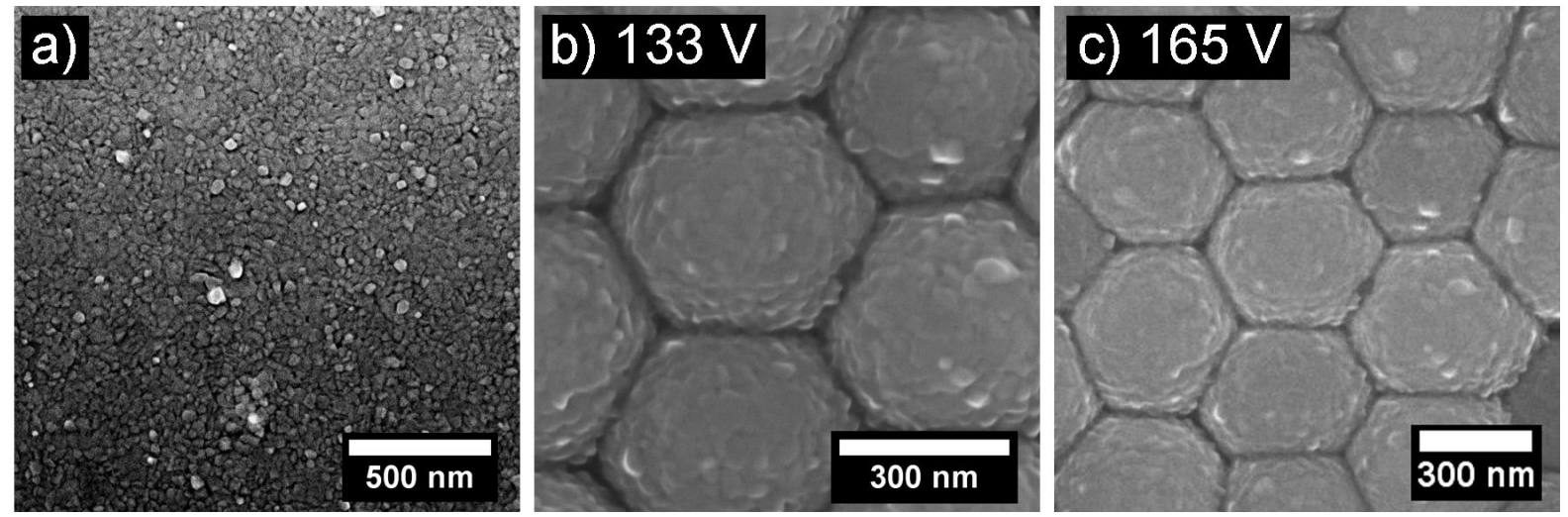

FIGURE 2. SEM images of $50 \mathrm{~nm}$ thick Al films on a) Non-texturized substrate (glass), b) and c) Texturized substrates with different anodizing voltages.
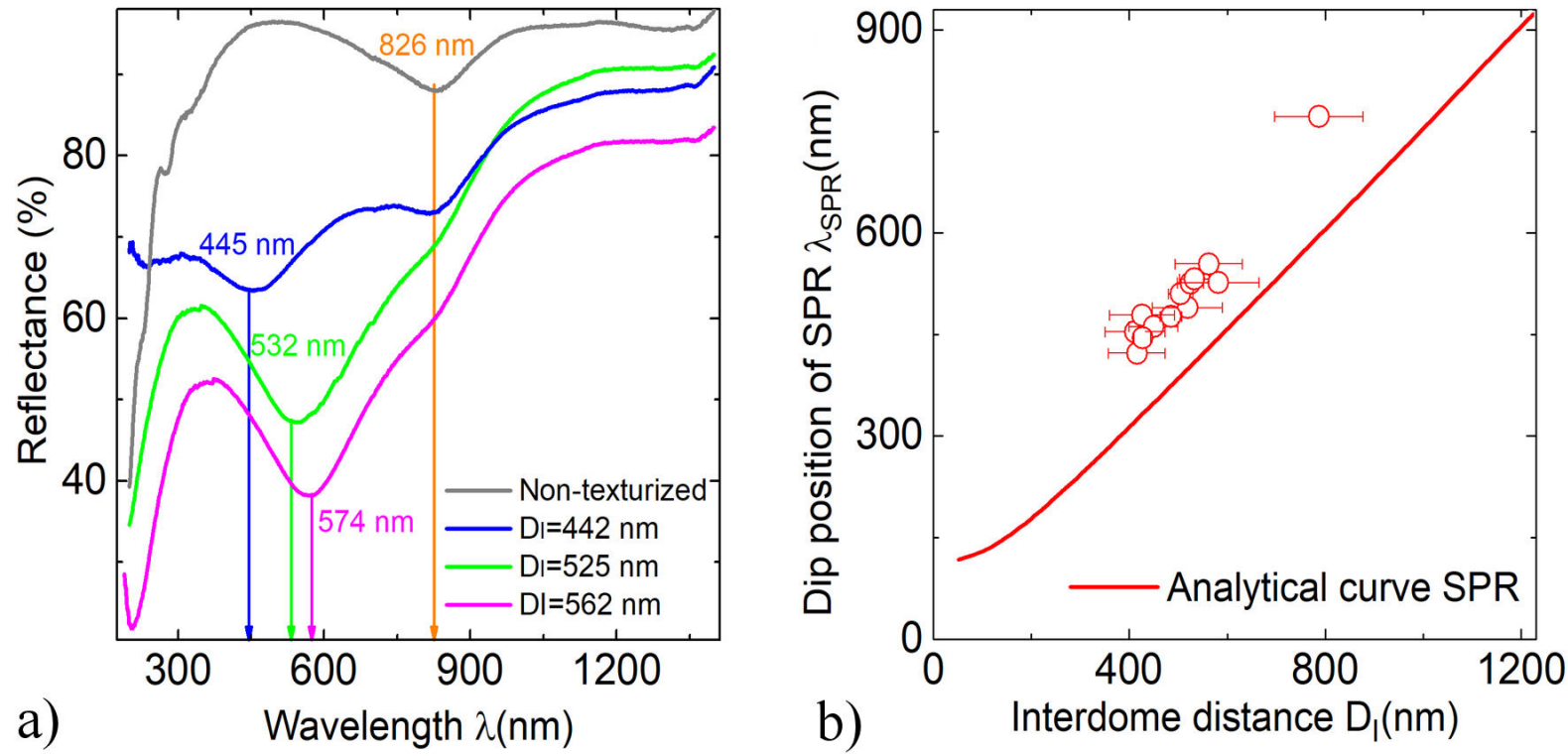

FIgURE 3. a) Reflectance spectra of Al-MF with different $D_{I}$. The results for the non-texturized Al film (gray curve) are shown for comparison. b) Experimental data obtained from Al-MF. The analytical curve of SPR (red curve) was performed using the model given by Eq. (1) with an incidence of $8^{\circ}$ and the optical constants for $\mathrm{Al}$ reported in [23].

where $\varepsilon_{m}$ and $\varepsilon_{d}$ are the dielectric functions of the metal and the dielectric (in our case air, $\varepsilon_{d}=1$ ), respectively; $\theta$ is the angle of incidence of the electromagnetic waves, $D_{I}$ is the period of the array (in our case the interdome distance) and $i, j$ the orders of dispersion. In Fig. 3b), experimental data corresponding to the dip position observed in the reflectance spectra of Al-MF as well as its respective theoretical curve obtained from Eq. (1) [20] are shown.

As shown in Fig. 3a), the position of the minima corresponding to SPR $\left(\lambda_{\mathrm{SPR}}\right)$ in the reflectance spectra depends on the dimensions of the $D_{I}$. In addition, another dip is observed in all samples at $826 \mathrm{~nm}$ related to IT in $\mathrm{Al}[25,26]$. It is worth mentioning that the theoretical model given by Fig. (1) reasonably follows the experimental results (Fig. 3b)). This behavior has also been observed in $\mathrm{Ag}$ and $\mathrm{Pb}$ metafilms [20]; which demonstrates the possibility of the excitation of surface plasmons in different texturized metals.
For measurements of $T$ as a function of the intensity of incident radiation, Al-MF were synthesized with $\lambda_{\mathrm{SPR}}=445$ $\mathrm{nm}$ and $\lambda_{\mathrm{SPR}}=532 \mathrm{~nm}$. According to the results shown in Fig. 4, the increases in $T$ are in the range of $16-204$ $\mathrm{K}$ considering the ambient $T(\sim 294 \mathrm{~K})$. For the case of the non-texturized $\mathrm{Al}$ film (Fig. 4a)), the maximum reached temperature was $\sim 316 \mathrm{~K}$ using an incident three wavelength of $650 \mathrm{~nm}\left(\lambda_{\text {inc }}=650 \mathrm{~nm}\right)$; while for Al-MF with $\lambda_{\mathrm{SPR}}=445$ $\mathrm{nm}$ and $\lambda_{\mathrm{SPR}}=532 \mathrm{~nm}$ (Figs. 4-b) and 4-c)), the reached temperatures were $\sim 432 \mathrm{~K}$ and $\sim 498 \mathrm{~K}$, respectively. In these last two cases, the increase in $T$ by radiation was greater compared to the non-texturized Al film. In particular, overheating occurs when Al-MF is illuminated with a wavelength close to or equal to its SPR, that is, when $\lambda_{\text {inc }} \approx \lambda_{\text {SPR }}$. Under these conditions, the SP decays in a non-radiative way, generating hot electrons [16] that transfer their energy to the phonons, thus increasing $T$ locally [5]. 


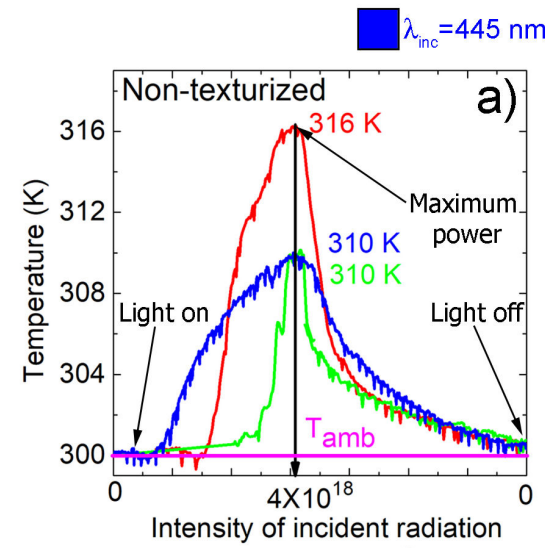

(Photons $\left./(\mathrm{s}) \mathrm{cm}^{2}\right)$
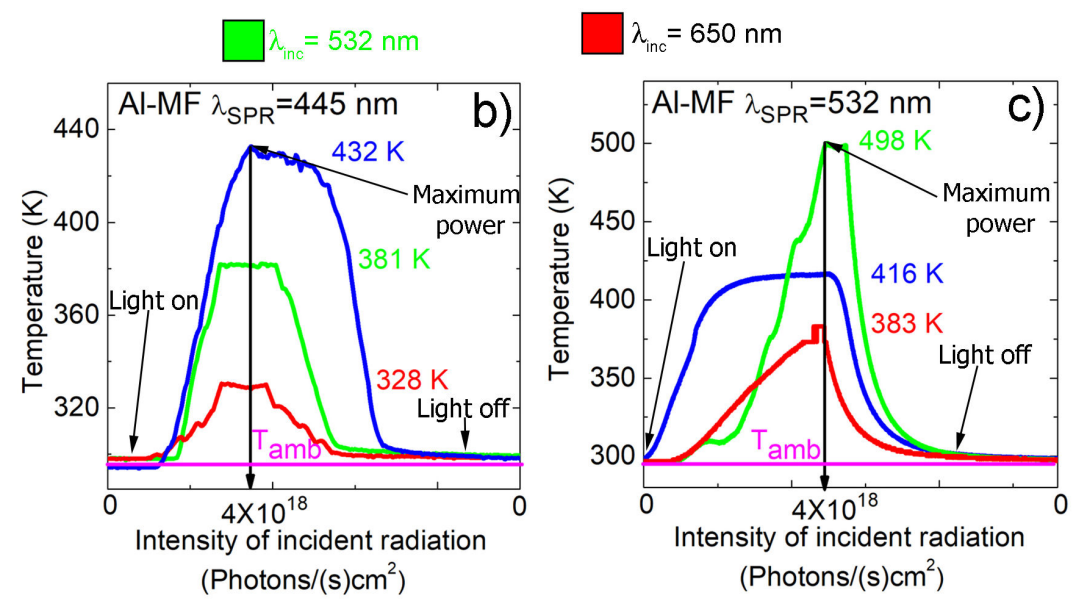

FIGURE 4. $T$ curves as a function of the intensity of incident radiation in a) Non-texturized Al film. Al-MF with b) $\lambda_{S P R}=445 \mathrm{~nm}$ and c) $\lambda_{S P R}=532 \mathrm{~nm}$. The intensity of light begins increasing from zero (Light on) up to a maximum intensity of $4 \times 10^{18}$ photons $/(\mathrm{s}) \mathrm{cm}^{2}$ (Maximum power), then it is decreased again to zero (Light off); the total process made in a time of 1 minute, such as is observed in the horizontal axis. The color of the curves corresponds to the shown wavelengths.
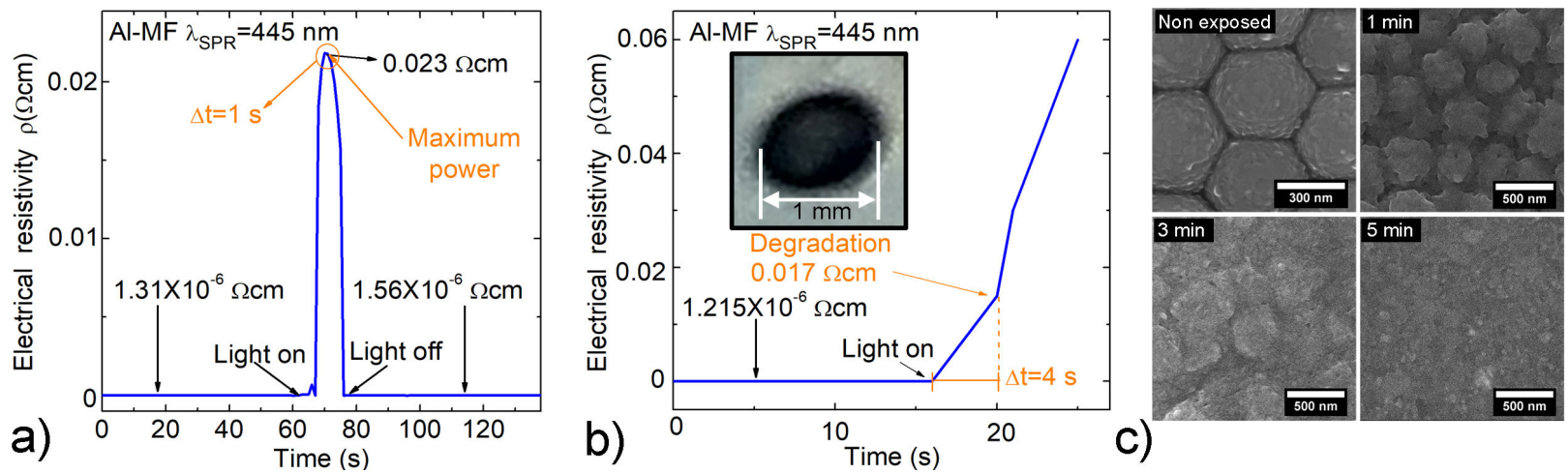

FIGURE 5. Al-MF $\rho$ curves with $\lambda_{\mathrm{SPR}}=445 \mathrm{~nm}$ irradiated with an intensity of $\sim 4 \times 10^{18}$ photons $/(\mathrm{s}) \mathrm{cm}^{2}$ and a) $t_{\mathrm{exp}}=1 \mathrm{~s}$ and b) $t_{\exp }>4 \mathrm{~s}$; the inset image belongs to the photograph of a sample damaged by local overheating.The samples were progressively irradiated (Light on) to the laser's maximum power and back to darkness (Light off) c) SEM images from the morphology evolution of an Al-MF irradiated at the different $t_{\exp }$ shown in the text.

Measurements of $\rho$ as a function of radiation exposure time $\left(t_{\exp }\right)$ were performed using an $\lambda_{\text {inc }}=\lambda_{\mathrm{SPR}}=445 \mathrm{~nm}$ with power of $4 \times 10^{18}$ photons $/(\mathrm{s}) \mathrm{cm}^{2}$. As shown in Fig. 5a), when $t_{\exp }$ is $\sim 1 \mathrm{~s}$ there is an increase in $\rho$ from $\sim 1.3 \times 10^{-6} \Omega \mathrm{cm}$ to $2.3 \times 10^{-2} \Omega \mathrm{cm}$. In this case, the Al-MF does not show notable deterioration; in addition, its $\rho$ returns approximately to the initial value $\left(\sim 1.5 \times 10^{-6} \Omega \mathrm{cm}\right)$ before being exposed to radiation. On the other hand, in Fig. 5b) it is observed that if the $t_{\exp }$ is greater than $1 \mathrm{~s}$, the value of $\rho$ continues to increase due to local overheating in the sample. In particular, at $t_{\exp }=4 \mathrm{~s}$, a change in the slope of $\rho$ is observed due to the degradation and subsequent evaporation of Al-MF (see inset Fig. 5b)); in this case, $\rho$ does not return to its initial value when irradiation is ceased.

Figure 5c) shows SEM images of the evolution of the morphology of an Al-MF with $\lambda_{\mathrm{SPR}}=445 \mathrm{~nm}$ when irradiated with an $\lambda_{\text {inc }}=445 \mathrm{~nm}$ at a power of $4 \times 10^{18}$ photons $/(\mathrm{s}) \mathrm{cm}^{2}$ to a different $t_{\text {exp. }}$. The change in its appearance (degradation) due to local overheating is clearly observed.
Locally, the temperatures reached by overheating due to non-radiative decay of SP are estimated to be $\sim 933 \mathrm{~K}$ (melting point of $\mathrm{Al}$ ), although it has been reported that the melting point of Al may decrease as the size of the particle decreases ( $\sim 3 \mathrm{~K}$ for a particle radius of $\sim 40 \mathrm{~nm}$ ) [27], in our case we estimate that the effect must be negligible since the thickness of our film is $50 \mathrm{~nm}$ and continuous. Recently, it has been shown that by the excitation of SP in Al nanoparticles, it is possible to achieve a $T$ of $\sim 975 \mathrm{~K}$ [15].

To qualitatively show the increase in $T$ in located areas, a drop of water of $1 \mathrm{~cm}$ in diameter was placed on an Al-MF with $\lambda_{\mathrm{SPR}}=445 \mathrm{~nm}$ and it was irradiated with an $\lambda_{\text {inc }}=445$ $\mathrm{nm}$ with $1 \mathrm{~mm}$ diameter at normal incidence, at a power of $\sim 4 \times 10^{18}$ photons $/(\mathrm{s}) \mathrm{cm}^{2}$. Figure 6 shows the experimental setup, where water vapor bubbles are observed in the area where the laser hits. These appear because temperatures above the boiling point of water are reached (see Fig. 4b)). This fact could be applied to water purification methods [28], to the development of plasmon-mediated nano heat sources 


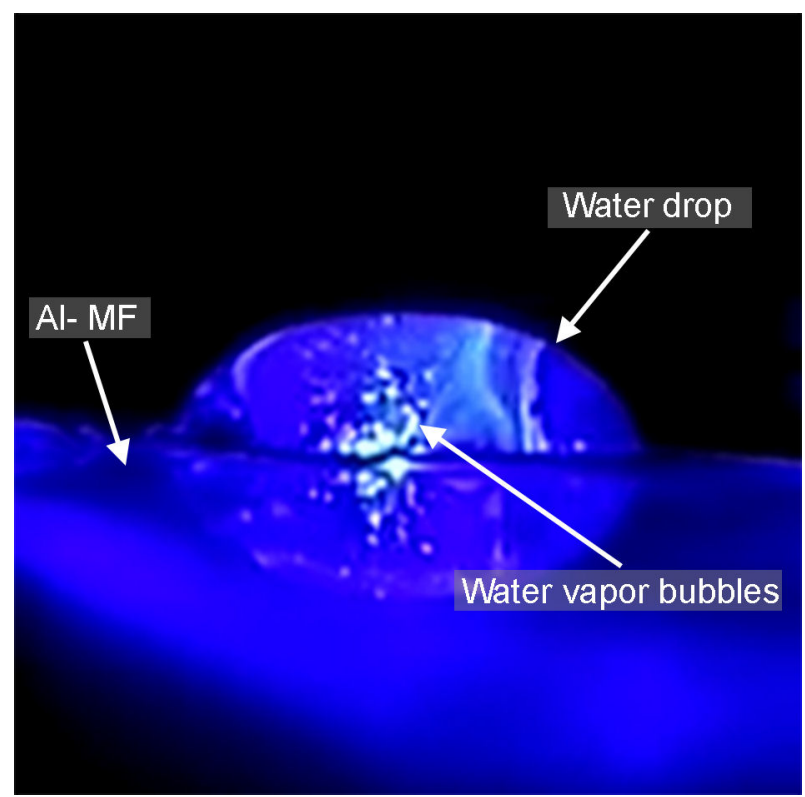

FIGURE 6. Water vapor bubbles induced by overheating in an AlMF with $\lambda_{\mathrm{SPR}}=445 \mathrm{~nm}$ when it is irradiated at normal incidence with a laser light source of $\lambda_{\text {inc }}=445 \mathrm{~nm}$.
[8], cancer treatments [5], or to generate and control microbubbles, since they are highly relevant in fundamental problems in thermodynamics in the study of local heating of fluids [9]. The following reference shows a video of the observed phenomenon [29]. When the water vapor bubbles are generated, a sound can be perceived, which we assign to a possible phenomenon of nanocavitation [9].

On the other hand, according to Figs. 5a) and 5b), the changes of $T$ in the Al-MF cause a considerable effect on the $\rho$, it is for this reason that measurements of $\rho(T)$ were carried out irradiating the samples under the same lighting conditions and low intensity $1 \times 10^{16}$ photons $/(\mathrm{s}) \mathrm{cm}^{2}$. Figure 7 shows the curves of $\rho(T)$ for a non-texturized film (reference sample) (Fig. 7a)) and Al-MF with $\lambda_{\mathrm{SPR}}=445 \mathrm{~nm}$ and $\lambda_{\mathrm{SPR}}=532 \mathrm{~nm}$ (Figs. 7b) and 7c), respectively).

In Fig. 7, it is observed that $\rho$ decreases when $T$ decreases in all cases, which is the typical behavior in metals [30]. But the linear behavior of $\rho$ as a function of $T$ below the Debye temperature $\left(T_{D}\right)$ is slightly modified (see the reference pink line). We attribute this behavior to the fact that the electron undergoes scattering due to the texturing present in the sam-
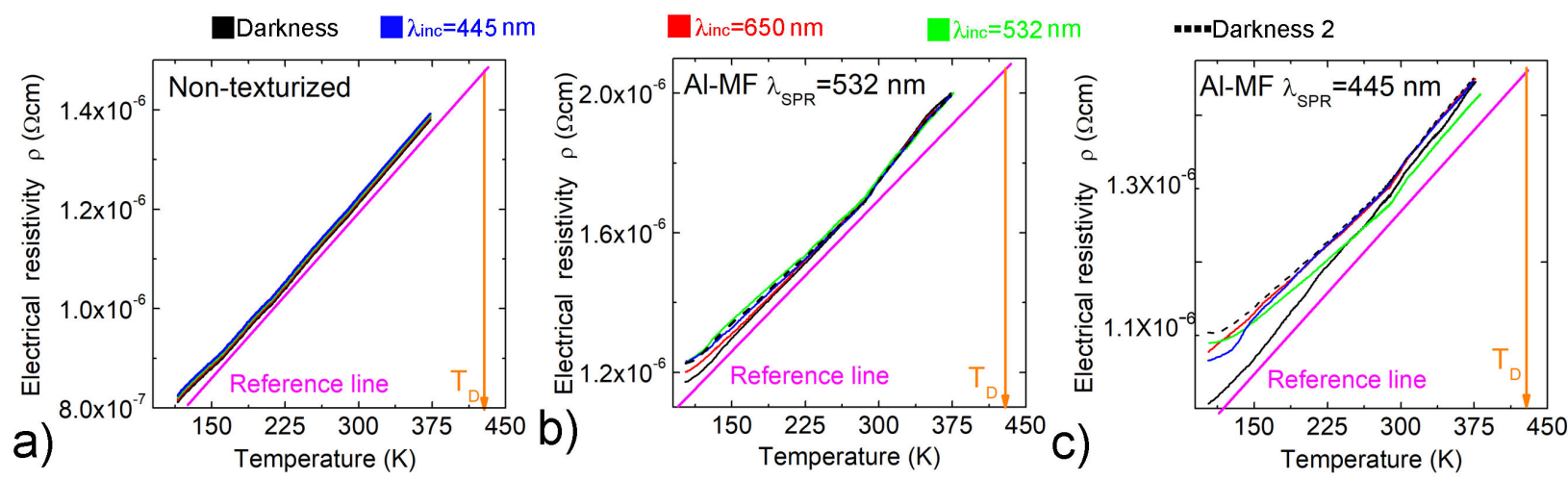

FIGURE 7. Curves of $\rho(T)$ in the dark and using different three wavelengths. a) Non-texturized film. Al-MF $\sim 50 \mathrm{~nm}$ thick with b) $\lambda_{\mathrm{SPR}}=445 \mathrm{~nm}$ and c) $\lambda_{\mathrm{SPR}}=532 \mathrm{~nm}$. In all cases, a reference line (pink line) and the Al Debye temperature $\left(T_{D}=428 \mathrm{~K}\right.$, orange line) [30] were placed. The continuous black curve is before the illumination process, the red, green, and blue curves correspond to the illumination with wavelenghts of 650,532 , and $445 \mathrm{~nm}$, respectively, while the dashed black curve is after the illumination process.

ples (Figs. 7b) and 7-c)). It is also observed that in a second measurement in darkness in the Al-MF (dashed curve) $\rho$ is slightly higher than the first one (black curve), possibly due to the deterioration caused by local heating when irradiated, even when the used light intensity is low; this effect is more noticeable at low temperatures.

\section{Conclusions}

The texturing of Al-MF allows a higher efficiency of absorption of light energy that is transformed into heat compared to non-texturized films. When Al-MF are illuminated with $\lambda_{\text {inc }}$ close to or equal to their $\lambda_{\text {SPR }}$, localized overheating occurs due to non-radiative deexcitation of surface plasmons and the creation of hot electrons that transfer their energy via phonons and finally heating the Al metafilm. This overheating causes a deterioration of the material and, consequently, a change in the $\rho$ of the Al-MF.

\section{Acknowledgments}

We thank Omar Novelo and Lourdes S. Bazán from Laboratorio Universitario de Microscopía Electrónica (LUMEUNAM) for SEM images, Miguel A. Canseco for his assistance in the UV-Vis equipment; Raúl Reyes, Carlos Ramos and Cain González for their technical support in the laboratory (all of them at IIM-UNAM). 
1. H. Raether, Surface Plasmons on Smooth and Rough Surfaces and on Gratings, (Springer, Berlin, 1988), pp. 4-7.

2. Mark L. Brongersma and Vladimir M. Shalaev, The case for plasmonics, Appl. Phys. 328 (210) 440, https://doi. org/10.1126/science.1186905

3. E. Ozbay, Plasmonics: Merging Photonics and Electronics at Nanoscale Dimensions, Science. 311 (206) 189, https : //doi.org/10.1126/science.1114849

4. A. Ramazani, F. Shayeganfar, J. Jalilian and N. X. Fang, Exciton-plasmon polariton coupling and hot carrier generation in two-dimensional $\mathrm{SiB}$ semiconductors: a first-principles study, Nanophotonics. 9 (2020) 337, https://doi.org/ 10.1515/nanoph-2019-0363

5. M. L. Brongersma, N. J. Halas and P. Nordlander, Plasmoninduced hot carriers science and technology, Nat. Nanotechnology. 10 (2015) 25, https://doi.org/10.1038/ nnano.2014.311

6. G. Baffou, F. Cichos and R. Quidant, Applications and challenges of thermoplasmonics, Nat. Mater. 19 (2020) 946, https://doi.org/10.1038/s41563-020-0740-6

7. X. Meng et al., Nanometals for Solar-to-Chemical Energy Conversion: From Semiconductor-Based Photocatalysis to Plasmon-Mediated Photocatalysis and Photo-Thermocatalysis, Adv. Mater. 28 (2016) 6781, https://doi.org/10. 1002 /adma.201600305

8. G. Baffou and R. Quidant, Thermo-plasmonics: using metallic nanostructures as nano-sources of heat, Laser Photonics Rev. 7 (2012) 1, https://doi.org/10.1002/lpor. 201200003

9. G. Baffou, J. Polleux, H. Rigneault and S. Monneret, SuperHeating and Micro-Bubble Generation around Plasmonic Nanoparticles under cw Illumination, J. Phys. Chem. C, 118 (2014) 4890, https : / / doi .org/10.1021/ jp411519k

10. H. U. Yang et al., Optical dielectric function of silver, Phys. Rev. B, 91 (2015) 23, https://doi.org/10.1103/ PhysRevB.91.235137

11. M. R. Beversluis, A. Bouhelier, and L. Novotny, Continuum generation from single gold nanostructures through nearfield mediated intraband transitions, Phys. Rev. B, 68 (2003) 115433, https://doi.org/10.1103/PhysRevB.68. 115433

12. D. Gérard and S. K. Gray, Aluminium plasmonics, Appl. Phys . 48 (2015) 18, https://doi.org/10.1088/ 0022-3727/48/18/184001

13. M. W. Knight, N. S. King, L. Liu, H. O. Everitt, P. Nordlander, and N. J. Halas, Aluminum for Plasmonics, ACS Nano. 8 (2014) 834, https://doi.org/10.1021/nn405495q

14. M. Schwind, B. Kasemo, and I. Zorić, Localized and Propagating Plasmons in Metal Films with Nanoholes, Nano Lett. . 13 (2013) 1743, https://doi.org/10.1021/nl400328x

15. M. Mutlu et al., Thermoplasmonic Ignition of Metal Nanoparticles, Nano Lett. . 18 (2018) 1699, https : //doi.org/10. 1021 /acs.nanolett. 7 b04739
16. B. Y. Zheng, H. Zhao, A. Manjavacas, M. McClain, P. Nordlander and N. J. Halas, Distinguishing between plasmon-induced and photoexcited carriers in a device geometry, Nat. Commun. 6 (2015) 1, https: / /doi.org/10.1038/ncomms 8797

17. M. T. Sheldon, J. van de Groep, A. M. Brown, A. Polman and H. A. Atwater, Plasmoelectric potentials in metal nanostructures, Nanophotonics. 346 (2014) 828, https : / / doi . org/ $10.1126 /$ science.1258405

18. Q. Guo et al., Christy, Efficient electrical detection of mid-infrared graphene plasmons at room temperature. Nat. Mater. 17 (2018) 986, https://doi.org/10.1038/ S41563-018-0157-7

19. R. González-Campuzano, M. E. Mata-Zamora, S. LópezRomero, and D. Mendoza, Excitation of plasmonic resonances within UV-Vis wavelength range using low-purity aluminum nanoconcave arrays, Appl. Phys. Lett 113 (2018) 221604, https://doi.org/10.1063/1.5059556

20. R. González-Campuzano, D. E. Martínez-Lara, and D. Mendoza, Lead plasmonics on texturized substrates: Pb metafilms, Appl. Phys. Lett 117 (2020) 032105, https://doi.org/ $10.1063 / 5.0016131$

21. R. González-Campuzano and D. Mendoza, Excitation of plasmons in self-ordered arrays of aluminum and silver nanocanves within UV-IR spectral range, J. Phys.: Conf. Sers. 1221 (2016) 012001, https://doi.org/10. 1088/1742-6596/1221/1/012001

22. C. A. Schneider, W. S. Rasband, and K. W. Eliceiri, NIH Image to ImageJ: 25 years of image analysis, Nat. Methods, 9 (2012) 671 https://doi.org/10.1038/nmeth.2089

23. Edward D. Palik, Hadbook of Optical Constants of Solids, Vol 2., Academic, (New York, 1985), pp. 395-401.

24. E. T. Papaioannou et al., Influence of the magnetic field on the plasmonic properties of transparent $\mathrm{Ni}$ anti-dot arrays, Appl. Phys. Lett , 19 (2011) 23867, https://10.1063/1. 4742931

25. H. Ehrenreich, H. R. Philipp, and B. Segall, Optical Properties of Aluminum, Phys. Rev. 132 (1963) 1918, https: //doi.org/10.1103/PhysRev.132.1918

26. A. J. Hughes, D. Jones, and A. H. Lettington, Calculation of the optical properties of aluminium, J. Phys. C: Solid State Phys. 2 (1968) 102, https://doi.org/10.1088/ 0022-3719/2/1/313

27. J. Sun and S.L. Simon, The melting behavior of aluminum nanoparticles, Thermochimica Acta 463 (2007) 32-40, https://doi.org/10.1016/j.tca.2007.07.007

28. L. Gomez, V. Sebastian, M. Arruebo, J. Santamaria and S. B. Cronin, Plasmon-enhanced photocatalytic water purification, Phys. Chem. 16 (2014) 15111 https://doi.org/ $10.1039 / \mathrm{C} 4 \mathrm{CP} 00229 \mathrm{~F}$

29. https://www youtube.com/watch?v=bv3aGLV jmA

30. C. Kittel, Introduction to Solid State Physics. University of California, Berkeley (John Wiley and Sons, Inc, 2005), pp. 265267. 